\title{
CT imaging of pulmonary embolism in patients with COVID-19 pneumonia: a retrospective analysis
}

\author{
Irene Espallargas ${ }^{1,2}$ (1) Juan José Rodríguez Sevilla ${ }^{3}$ • Diego Agustín Rodríguez Chiaradía 4,5,6,7 . Antonio Salar ${ }^{3,5}$. \\ Guillem Casamayor $^{8}$. Judit Villar-Garcia ${ }^{9,5}$. Anna Rodó-Pin ${ }^{4,5,6,7}$. Salvatore Marsico ${ }^{1}$. Santiago Carbullanca ${ }^{1}$. \\ Diego Ramal $^{1}$ - Luis Alexander del Carpio ${ }^{1}$. Ángel Gayete ${ }^{1}$ - José María Maiques ${ }^{1}$ - Flavio Zuccarino ${ }^{1,10}$
}

Received: 24 April 2020 / Revised: 8 September 2020 / Accepted: 15 September 2020 / Published online: 22 September 2020

(C) European Society of Radiology 2020

\begin{abstract}
Objectives To describe imaging and laboratory findings of confirmed PE diagnosed in COVID-19 patients and to evaluate the characteristics of COVID-19 patients with clinical PE suspicion. Characteristics of patients with COVID-19 and PE suspicion who required admission to the intensive care unit (ICU) were also analysed.

Methods A retrospective study from March 18, 2020, until April 11, 2020. Inclusion criteria were patients with suspected PE and positive real-time reverse-transcription polymerase chain reaction (RT-PCR) for SARS-CoV-2. Exclusion criteria were negative or inconclusive RT-PCR and other chest CT indications. CTPA features were evaluated and severity scores, presence, and localisation of PE were reported. D-dimer and IL-6 determinations, ICU admission, and previous antithrombotic treatment were registered.

Results Forty-seven PE suspicions with confirmed COVID-19 underwent CTPA. Sixteen patients were diagnosed with PE with a predominant segmental distribution. Statistically significant differences were found in the highest D-dimer determination in patients with PE and ICU admission regarding elevated IL-6 values.

Conclusion PE in COVID-19 patients in our series might predominantly affect segmental arteries and the right lung. Results suggest that the higher the D-dimer concentration, the greater the likelihood of PE. Both assumptions should be assessed in future studies with a larger sample size.

Key Points

- On CT pulmonary angiography, pulmonary embolism in COVID-19 patients seems to be predominantly distributed in segmental arteries of the right lung, an assumption that needs to be approached in future research.

- Only the highest intraindividual determination of D-dimer from admission to CT scan seems to differentiate patients with pulmonary embolism from patients with a negative CTPA. However, interindividual variability calls for future studies to establish cut-off values in COVID-19 patients.

- Further studies with larger sample sizes are needed to determine whether the presence of PE could increase the risk of intensive care unit (ICU) admission in COVID-19 patients.
\end{abstract}

Irene Espallargas

igespallargas@gmail.com

1 Department of Radiology, Hospital del Mar, Passeig Maritim 23-25, 08003 Barcelona, Spain

2 Department of Radiology, Hospital Germans Trias i Pujol, Carretera de Canyet S/N, 08916 Badalona, Spain

3 Department of Hematology, Hospital del Mar, Passeig Maritim 23-25, 08003 Barcelona, Spain

4 Pulmonology Department, Hospital del Mar, Passeig Maritim 23-25, 08003 Barcelona, Spain

5 Institut Hospital del Mar d'Investigacions Mèdiques (IMIM), Dr. Aiguader, 88, 08003 Barcelona, Spain
6 Universitat Pompeu Fabra (UPF), Dr. Aiguader, 80, 08003 Barcelona, Spain

7 CIBERES, (ISCIII), Calle de Melchor Fernández Almagro, 3, 28029 Madrid, Spain

8 Department of Emergency Medicine, Hospital Germans Trias i Pujol, Carretera de Canyet S/N, 08916 Badalona, Spain

9 Department of Infectious Diseases, Hospital del Mar, Passeig Maritim 23-25, 08003 Barcelona, Spain

10 Department of Radiology, Hospital Sant Joan de Déu, Passeig de Sant Joan de Déu, 2, 08950 Esplugues de Llobregat, Barcelona, Spain 
Keywords Computed tomography angiography · Pulmonary embolism · COVID- $19 \cdot$ Fibrin fragment D · Intensive care units

\author{
Abbreviations \\ COVID-19 Coronavirus infectious disease 2019 \\ RT-PCR Reverse-transcription polymerase \\ chain reaction \\ CTPA CT pulmonary angiography \\ PE Pulmonary embolism \\ ICU Intensive care unit \\ SARS-CoV-2 Severe acute respiratory syndrome \\ coronavirus 2
}

\section{Introduction}

The COVID-19 pandemic began in Wuhan (Hubei, China) in December 2019 and rapidly spread around the world. At the beginning of April 2020, there were over 100,000 confirmed cases and more than 10,000 deaths in Spain. Radiological literature has until now focused on non-contrast computed tomography (CT) findings to describe the probability of infection [1] due to limitations of sensitive real-time reverse-transcription polymerase chain reaction (RT-PCR) results [2, 3]. However, the elevation of $\mathrm{D}$-dimer has been reported in patients infected with the novel coronavirus [4], which, in our institution, has led to an increase in the number of CT pulmonary angiography (CTPA) requests with suspected pulmonary embolism (PE). D-dimers are able to rule out deep vein thrombosis and PE in outpatients with low or intermediate clinical probability of PE. However, unless specific cut-off levels are used, its usefulness in hospitalised patients with suspected thromboembolism is less established. Based on a retrospective study, D-dimers above $2000 \mu \mathrm{g} / \mathrm{L}$ were predictive of the presence of PE independently of the clinical score [5]. Besides, as D-dimer levels increase for around 7 days during the PE event, late evaluation may lead to underestimated determination [6]. The aim of this study is to describe the imaging and laboratory findings of confirmed PE diagnosed in COVID-19 patients. We have evaluated the characteristics of COVID-19 patients, with clinical suspicion of PE, who had had confirmatory CTPA and compared them to those who had not. In addition, we also analysed the characteristics of patients with COVID19 and PE suspicion who required admission to the intensive care unit (ICU).

\section{Materials and methods}

The study was approved by the Clinical Research Ethical Committee of the Parc de Salut Mar, which due to the exceptionality of the pandemic emergency waived the informed consent. A retrospective study was performed to analyse CT scans and laboratory of COVID-19 patients admitted to our hospital.

\section{Clinical evaluation}

Nine hundred and nineteen patients (804 laboratoryconfirmed SARS-CoV-2 and 115 pending result) with clinical COVID-19 were hospitalised in our institution during the time that this study data was registered. Due to sensitivity limitations of RT-PCR (around 70\% in the diagnostic kit used in our hospital), a situation already described [2, 3], and delays in obtaining results, clinical assessment was essential in the initial diagnosis of COVID-19 pneumonia (sudden onset of dry cough, fever, or shortness of breath [4]). Inclusion criteria were contrast-enhanced studies with CTPA protocol in adult patients with elevated clinical suspicion of PE and both positive screening and confirmatory RT-PCR for SARS-CoV-2. Exclusion criteria were negative or inconclusive RT-PCR, even when clinical and imaging findings were consistent with viral pneumonia, and other reasons for CT request other than PE suspicion. The CT studies were searched for in the electronic database from March 18 to April 11, while the clinical and laboratory data were collected through available electronic medical records. Time from the onset of the symptoms to the CT performance was collected under the name of time to PE (TTP).

Severe cases of COVID-19 are associated with increased IL-6 levels [7]. Cytokines are fundamental in managing immunological and severe reactions; among them, IL-6 is vital on account of its pleiotropic impacts [8]. A recent metaanalysis of the impact of IL-6 as disease progression predictor was performed; presenting evidence that circulating IL-6 levels are firmly connected to the severity of COVID-19 infection [9]. On the other hand, increased expression of IL-6 has correlated with an increase of the incidence of deep vein thrombosis [10]. On behalf of this evidence, measuring IL-6 levels was considered a standard of care for COVID-19 patients in our centre, not only in the follow-up of the COVID19 patient status but also in the consideration of antiinterleukin-16 agents (tocilizumab, sarilumab, siltuximab) [10]. The first determination of IL-6 after admission was collected for this study, as it would not be influenced by COVID19 treatments. Regarding D-dimer testing, initial values on admission and immediately before CT were registered. The highest D-dimer testing before the scan was also collected to avoid underestimation due to late evaluation.

Anticoagulant therapy before the CT examination and its doses were also registered since the intrinsic SARS-CoV-2 risk of disseminated intravascular coagulation and venous thromboembolism has led to the recommendation of 
anticoagulant treatment by expert consensus [11, 12]. In our centre, anticoagulant strategy in patients with COVID-19 has evolved during the pandemic. In the first period, all patients with no anticoagulation contraindications received prophylaxis with low molecular weight heparin at standard doses recommended for medically ill patients (enoxaparin $40 \mathrm{mg}$ per day subcutaneously). In the second period, patients who had a D-dimer above $2000 \mathrm{mcg} / \mathrm{L}$ received intermediate doses of low molecular weight heparin (enoxaparin $1 \mathrm{mg} / \mathrm{kg}$ per day subcutaneously), while the other patients had standard dose prophylaxis.

Admission to the ICU for COVID-19 patients in our institution included clinical (tachypnoea, tachycardia, persistent hypotension, Glasgow coma scale $\leq 14$, persistent respiratory failure under Venturi mask with $50 \% \mathrm{FIO}_{2}$, and abnormal work of breathing) and/or laboratory criteria $(\mathrm{pH}<7.3$, $\mathrm{HCO} 3<15 \mathrm{mmol} / \mathrm{L}$ or $\mathrm{PaCO}_{2}>60 \mathrm{mmHg}$, lactate $>3.5 \mathrm{mmol} / \mathrm{L}$, persistent renal failure, and disseminated intravascular coagulation).

\section{Imaging and interpretation}

CTPA examinations were obtained in a multidetector CT scanner (Discovery CT750 HD, GE Healthcare) by using a dual-energy CTPA protocol (Gemstone Spectral Imaging GSI), with the following parameters: tube voltage $80-$ $140 \mathrm{kV}, 340 \mathrm{~mA}$, pitch $=0.984$, rotation time $=0.5 \mathrm{~s}$, field of view $=512 \mathrm{~mm} \times 512 \mathrm{~mm}, 64 \times 0.625-\mathrm{mm}$ detector configuration, and slice thickness $=1.25 \mathrm{~mm}$. The $\mathrm{CT}$ acquisition was performed using automatic bolus-tracking technique (region of interest located at the pulmonary artery with a trigger threshold of $150 \mathrm{HU}$ ) after a weight-based protocol injection of 50-70 mL of nonionic iodinated contrast media (Omnipaque 350, GE Healthcare) at a flow rate of $4 \mathrm{~mL} / \mathrm{s}$, followed by a $25-\mathrm{mL}$ saline flush. DICOM data was transferred to a PACS workstation (Centricity Universal Viewer v.6.0, GE Medical Systems), for multiplanar reconstruction and evaluation, in both lung and mediastinal windows, of monochromatic CT angiographic images. Iodine maps were analysed with dedicated software (GSI viewer, AW server 2.2, GE Healthcare) and used to improve accuracy for distal perfusion defects [13]. Homogeneous lung perfusion did not exclude the meticulous revision of pulmonary arteries on CTPA images. Studies were considered non-diagnostic whenever breathing or streak artefacts impaired segmental, lobar, and main pulmonary branches evaluation. Subsegmental arteries were carefully examined before and after iodine map inspection but a lack of complete visualisation was reported as negative CTPA. Our thoracic imaging section members (senior radiologists with 12 and 29 years of experience) reviewed all cases and resolved discrepancies by consensus.

$\mathrm{PE}$ detection and location were reported and grouped into central and lobar PE or segmental and subsegmental PE for analysis. The following features were used to establish the pattern of COVID-19 pneumonia: the presence of multiple bilateral foci of ground-glass opacities (GGO), lower lobe predominance with peripheral distribution that could be associated with crazy-paving pattern, peripheral consolidation, air bronchograms, perilobular pattern, and/or reverse "halo" sign $[14,15]$. Patients were defined as positive when the pattern was consistent with COVID-19 pneumonia and isolated as such in wards established for this purpose.

All CT disease patterns were quantified with a score from 1 to 4 in mild (1), when there were up to 3 focal pure GGO of less than $3 \mathrm{~cm}$ in maximum diameter; moderate-severe (2) when there were more than 3 focal GGO or maximum diameter superior to $3 \mathrm{~cm}$; moderate-severe (3) in focal GGO mixed with early consolidation; and severe (4) when there were diffuse GGO or consolidation and signs of architectural distortion [15]. As reviewed by the British Society of Thoracic Imaging, differences between moderate and severe disease have a great amount of subjectivity which may lead to low interobserver agreement.

\section{Statistical analysis}

Statistical analysis was performed with the IBM SPSS Statistics version 23.0 software (IBM). Continuous data were expressed as median with its interquartile range and full range and were tested for normality using the Kolmogorov-Smirnov test. Non-normally distributed variables were compared with the Mann-Whitney $U$ test. Categorical variables were described as number (percentage) and compared with Fisher's exact test. Missing data implied patient exclusion for that variable analysis.

A $p$ value of less than 0.05 was considered statistically significant.

\section{Results}

Forty-seven PE suspicions meeting the inclusion criteria for COVID-19 pneumonia were registered in the Diagnostic Imaging Department and underwent CTPA scan. None of these studies was considered non-diagnostic. There were 17 (36\%) females and $30(64 \%)$ males, with a median age of 65 years (IQR, 54-73, range 30-94). In 45 patients (96\%), both RT-PCR and CTPA were concordant for COVID-19. Two patients had positive RT-PCR with non-COVID-19 pulmonary findings on the CT scan. One of these two normal CT corresponded to a patient in "early stage" ( 3 days after the onset of symptoms). The severity of the pulmonary findings was distributed as such: $2(4.25 \%)$ normal CT scans, two $(4.25 \%)$ mild, three $(6.4 \%)$ moderate-severe, eleven $(23.4 \%)$ moderate-severe, and 29 (61.7\%) severe cases (Fig. 1). Twenty-three patients $(49 \%)$ were admitted to the ICU. 
Fig. 1 Images ordered by score from the upper left to bottom right in mild $(\mathbf{a}=$ score 1$)$, moderatesevere $(\mathbf{b}=$ score 2$)$, moderatesevere $(\mathbf{c}=$ score 3$)$, and severe $(\mathbf{d}=$ score 4$)$

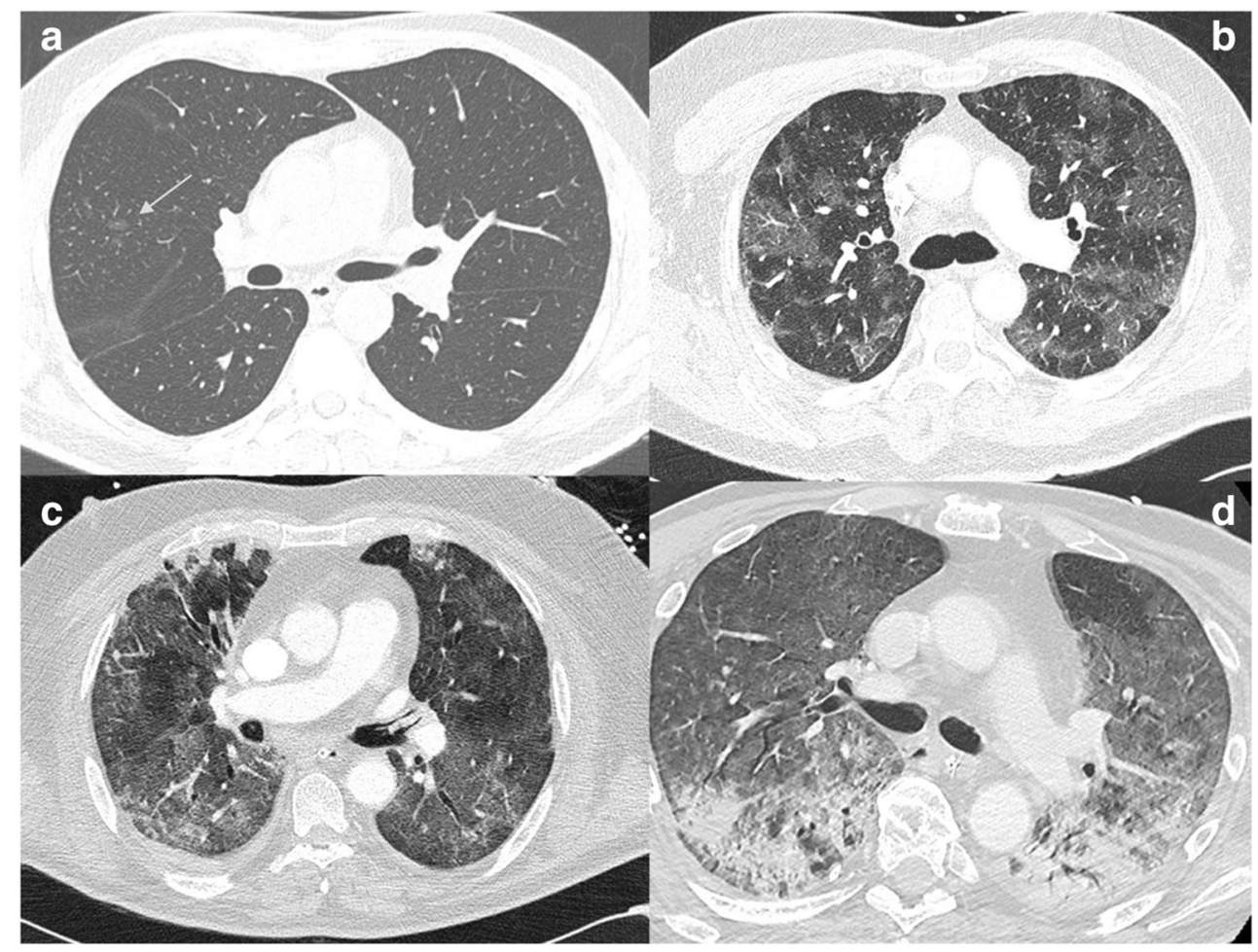

Thirty-six patients (77\%) received enoxaparin treatment before the CT examination: prophylactic dosage $40 \mathrm{mg} /$ day $(n=$ $18,50 \%)$, intermediate doses $\mathrm{mg} / \mathrm{kg} / \mathrm{day}(n=17,47.2 \%)$, and full-dose regimen $\mathrm{mg} / \mathrm{kg} / 12 \mathrm{~h}(n=1,2.8 \%)$. Sixteen patients (34\%) were diagnosed with PE with the clinicoradiologic characteristics being shown in Table 1 . Three patients (18.75\%) had central PE and 4 patients (25\%) had lobar PE, while 9 patients $(56.25 \%)$ had purely segmental $(5,31.25 \%)$ or subsegmental (4, 25\%) PE (Figs. 2, 3, 4, and 5). The right lung was involved in 15 cases $(93.75 \%)$ and in 9 cases (56.25\%), the left lung was affected. Three patients had CT signs of right cardiac overload, with one of them having central filling defects and the other two lobar or segmental arteries affected (Fig. 3). The median time to PE (TTP) was 16 days

Table 1 Confirmed PE in COVID-19 patients

\begin{tabular}{|c|c|c|c|c|c|c|c|c|c|c|}
\hline & \multirow[b]{2}{*}{ Gender } & \multirow[b]{2}{*}{ Age (y) } & \multicolumn{3}{|c|}{ D-dimer $(\mu \mathrm{g} / \mathrm{L})$} & \multirow[t]{2}{*}{ TTP (days) } & \multirow[t]{2}{*}{$\mathrm{PE}$} & \multirow[t]{2}{*}{ Sites of PE } & \multirow{2}{*}{$\begin{array}{l}\text { Anticoagulant } \\
\text { therapy } \\
\text { prior to CT }\end{array}$} & \multirow[t]{2}{*}{$\mathrm{ICU}$} \\
\hline & & & Initial & Highest & DDCT & & & & & \\
\hline Patient 1 & $\mathrm{M}$ & 62 & 310 & 4330 & 2330 & 10 & Lobar + segmental & $R U L, R L L, L U L, \mathrm{LLL}$ & Prophylaxis & Yes \\
\hline Patient 2 & $\mathrm{~F}$ & 76 & 1420 & 35,200 & 32,140 & 21 & Central & Bilateral & Prophylaxis & Yes \\
\hline Patient 3 & $\mathrm{~F}$ & 48 & 10,050 & 19,680 & 4700 & 12 & Segmental & RLL, RUL & Prophylaxis & Yes \\
\hline Patient 4 & M & 73 & 650 & 33,600 & 20,280 & 14 & Segmental & RUL, LUL, LLL, lingula & Prophylaxis & Yes \\
\hline Patient 5 & M & 78 & $>35,200^{*}$ & $>35,200 *$ & $>35,200$ & 2 & Central & Bilateral & No & No \\
\hline Patient 6 & $\mathrm{~F}$ & 34 & 2140 & $5930 *$ & 5930 & 17 & Segmental & RLL & Prophylaxis & Yes \\
\hline Patient 7 & $\mathrm{~F}$ & 69 & 540 & 5140 & 4360 & 14 & Lobar + segmental & $L L L$, LUL, RLL, RUL & Intermediate & Yes \\
\hline Patient 8 & $\mathrm{~F}$ & 72 & 2350 & 31,550 & 12,160 & 15 & Central & Bilateral & Intermediate & No \\
\hline Patient 9 & $\mathrm{~F}$ & 59 & 1320 & 6120 & 5620 & 12 & Lobar + segmental & LUL, LLL, RUL, RLL, ML & Prophylaxis & No \\
\hline Patient 10 & M & 69 & 3510 & 3510 & 2030 & 20 & Subsegmental & RLL & Prophylaxis & No \\
\hline Patient 11 & M & 56 & 350 & 35,200 & 9900 & 29 & Subsegmental & RUL & Intermediate & Yes \\
\hline Patient 12 & M & 59 & 360 & $15710^{*}$ & 15,710 & 17 & Subsegmental & LLL & Intermediate & Yes \\
\hline Patient 13 & M & 48 & 1070 & 12,950 & 3790 & 18 & Subsegmental & RUL & Intermediate & Yes \\
\hline Patient 14 & $\mathrm{~F}$ & 94 & $6570 *$ & $6570 *$ & 6570 & 3 & Segmental & ML, RUL, LLL & No & No \\
\hline Patient 15 & M & 71 & 390 & 35,200 & 4670 & 25 & Segmental & RUL & Intermediate & Yes \\
\hline Patient 16 & M & 58 & 2100 & 23,970 & 10,840 & 22 & Lobar & RLL & Intermediate & Yes \\
\hline
\end{tabular}

$D D C T$, D-dimer prior to the CT. $y$, year; $M$, male; $F$, female; $T T P$, time To PE; $P E$, acute pulmonary embolism; $I C U$, intensive care unit; $R L L$, right lower lobe; $R U L$, right upper lobe; $M L$, median lobe; $L U L$, left upper lobe; $L L L$, left lower lobe. Sites of PE in italics are the lobar affected arteries. * D-dimer values are the same from the DDCT 


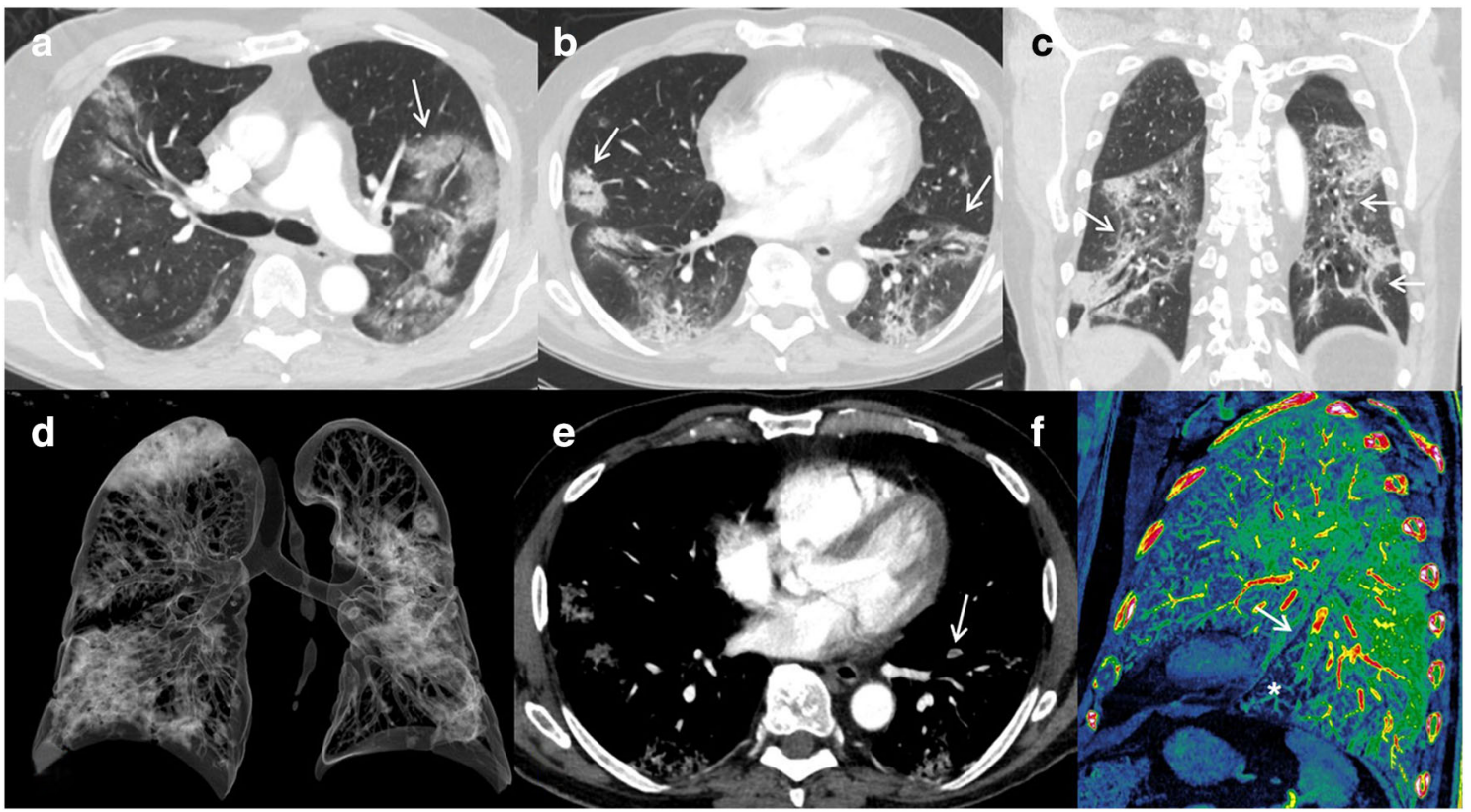

Fig. 2 Segmental left lower lobe PE over a severe lung involvement. CTPA, with lung window $(\mathbf{a}, \mathbf{b}, \mathbf{c})$ and volume rendering (d) images, shows extensive lung involvement (score 4) with typical findings as reverse halo sign (a, arrow), bilateral peripheral GGO and consolidations with perilobular distribution (b, arrows), and

(IQR 12-20.75, range 2-29). The patient with the lowest TTP ( 2 days) had been discharged from a pacemaker insertion 2 days before the onset of symptoms.

In COVID-19 patients with PE, the highest intraindividual D-dimer values were statistically significant when compared with the non-PE group $(p=0.015)$. No other differences architectural distortion with peripheral sparing (c, arrows). We can also appreciate (e) a small peripheral thrombus (arrow) in a segmental artery of the left lower lobe. Sagittal iodine map image (f) allows us to define segmental vessel obstruction (arrow) and peripheral hypoperfusion (asterisk)

between groups regarding gender, age, the rest of D-dimer determinations, lung disease score, initial IL-6, anticoagulant therapy, or anticoagulant doses prior to the $\mathrm{CT}$ were statistically significant. However, a difference in the means of initial IL-6 in PE patients $(125.08 \mathrm{pg} / \mathrm{mL})$ versus non-PE patients $(534.82 \mathrm{pg} / \mathrm{mL})$ was seen. There were no differences in the

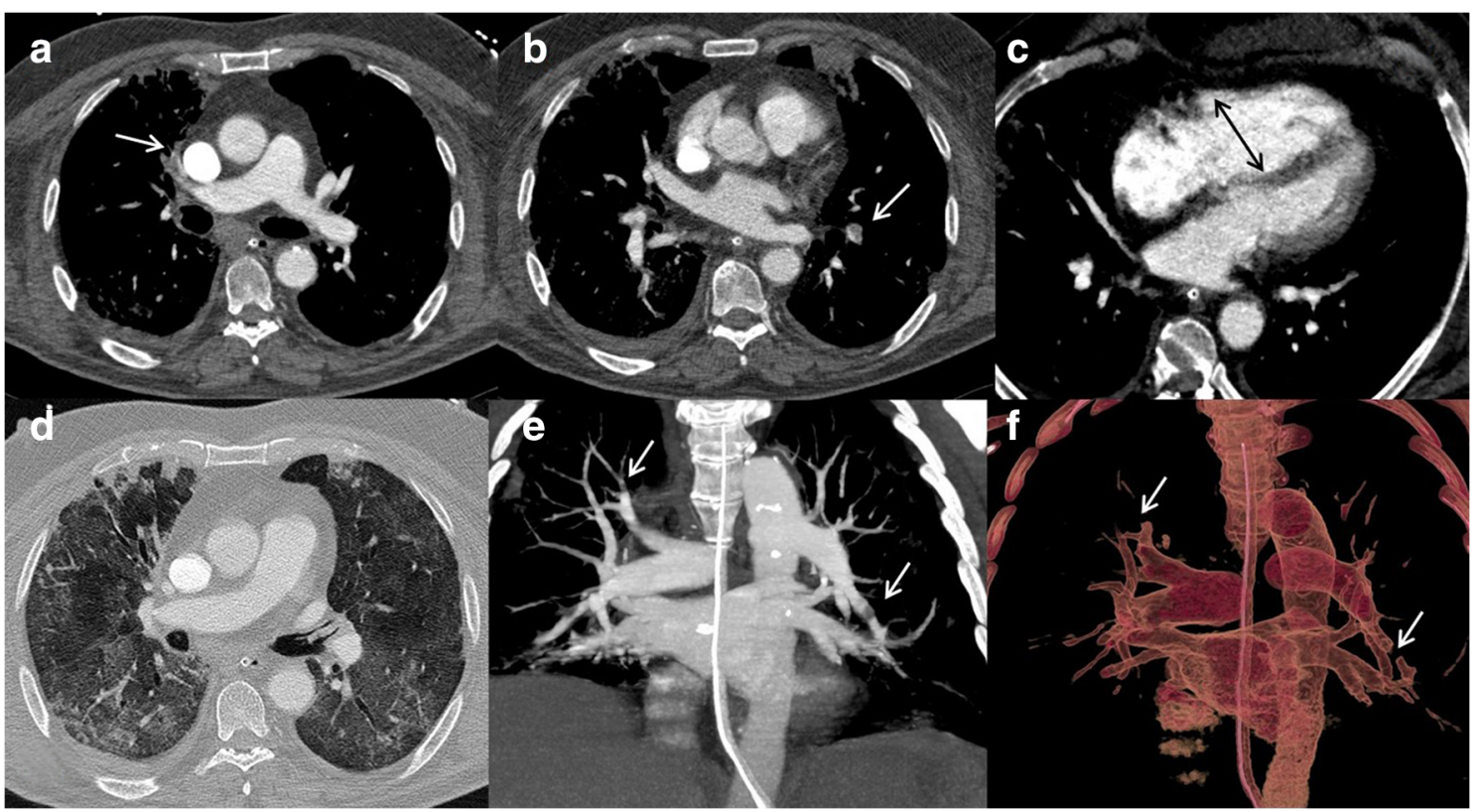

Fig. 3 Segmental left lower lobe and right upper lobe PE. Segmental bilateral embolisms (arrows) can be appreciated in axial (a, b) and oblique MIP and VR images (e, f) over a moderate-severe (score 3) pulmonary involvement (d). Signs of right cardiac overload (black arrow) with interventricular septum shifting towards the left ventricle are shown in $\mathbf{c}$ 

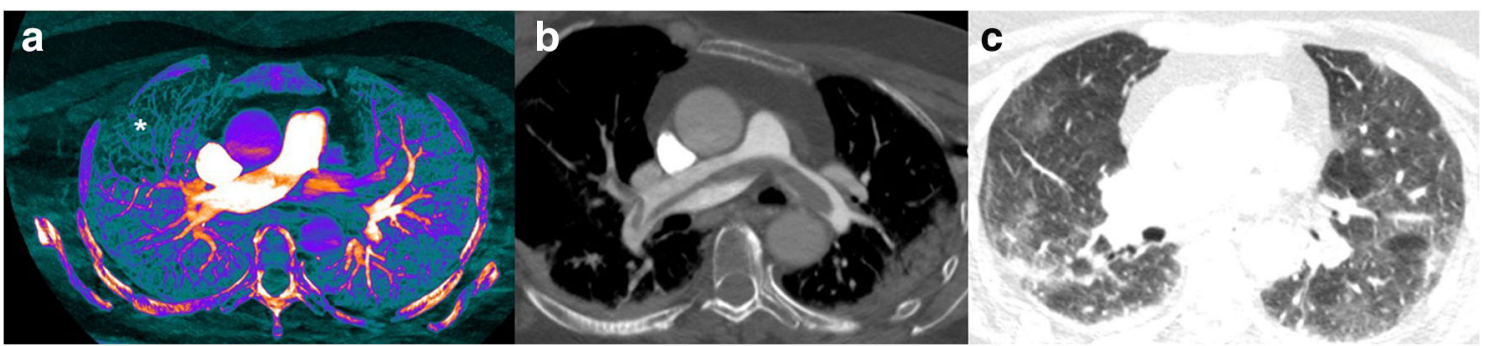

Fig. 4 Saddle pulmonary embolism. Saddle pulmonary embolism can be appreciated in axial (b) mediastinal window, over a moderate-severe (score 3) pulmonary involvement (c). Iodine map image depicts a hypoperfusion area (a; asterisk) in the right lung

sites of PE with respect to either D-dimer determinations or previous antithrombotic treatment.

Even though $11 / 16$ patients $(68.8 \%)$ with PE were admitted to the ICU compared with $12 / 31$ (38.7\%) in the negative CTPA group, there were no statistically significant differences in the 2-sided Fisher's exact test $(p=0.069)$ regarding ICU admission. On analysing the ICU admission data in suspected PE patients, statistically significant differences were seen in anticoagulant therapy prior to $\mathrm{CT}(p=0.036)$, as patients in the ICU were more likely to receive antithrombotic treatment and also higher dosages $(p=0.007)$. Significant differences were also seen in initial IL-6 $(p<0.001)$, being higher in admitted ICU patients (mean $565.8 \mathrm{pg} / \mathrm{mL}$ versus $236.61 \mathrm{pg} / \mathrm{mL}$ in non-ICU admitted patients). Differences were found regarding the highest D-dimer determinations $(15,195.22 \mu \mathrm{g} / \mathrm{L}$ in ICU admitted patients vs $9541.3 \mu \mathrm{g} / \mathrm{L}$ in non-ICU admitted patients), although they were not statistically significant ( $p=$ 0.084 ). There were no statistically significant differences regarding lung disease score; however, a slightly higher score was seen in ICU-admitted patients (mean 3.13 in non-ICU patients versus 3.57 in ICU patients).

\section{Discussion}

CT findings in COVID-19 patients were very similar to the ones described in other countries, with predominantly peripheral ground-glass foci that evolved into consolidation and architectural distortion $[3,14,16]$. Lung disease scores were significantly high, which correlates with severe illness in patients with PE suspicion. There was an equal number of unilateral and bilateral PE, emphasising a majority of segmental PE (Table 1). The predominant involvement of the right lung, especially its upper lobe, is also interesting, while the median lobe and the lingula seem to have rarely been affected.

COVID-19 patients have difficulties holding their breath during CTPA acquisition, which diminishes their eligibility for peripheral PE. Dual-energy CTPA protocol may help to

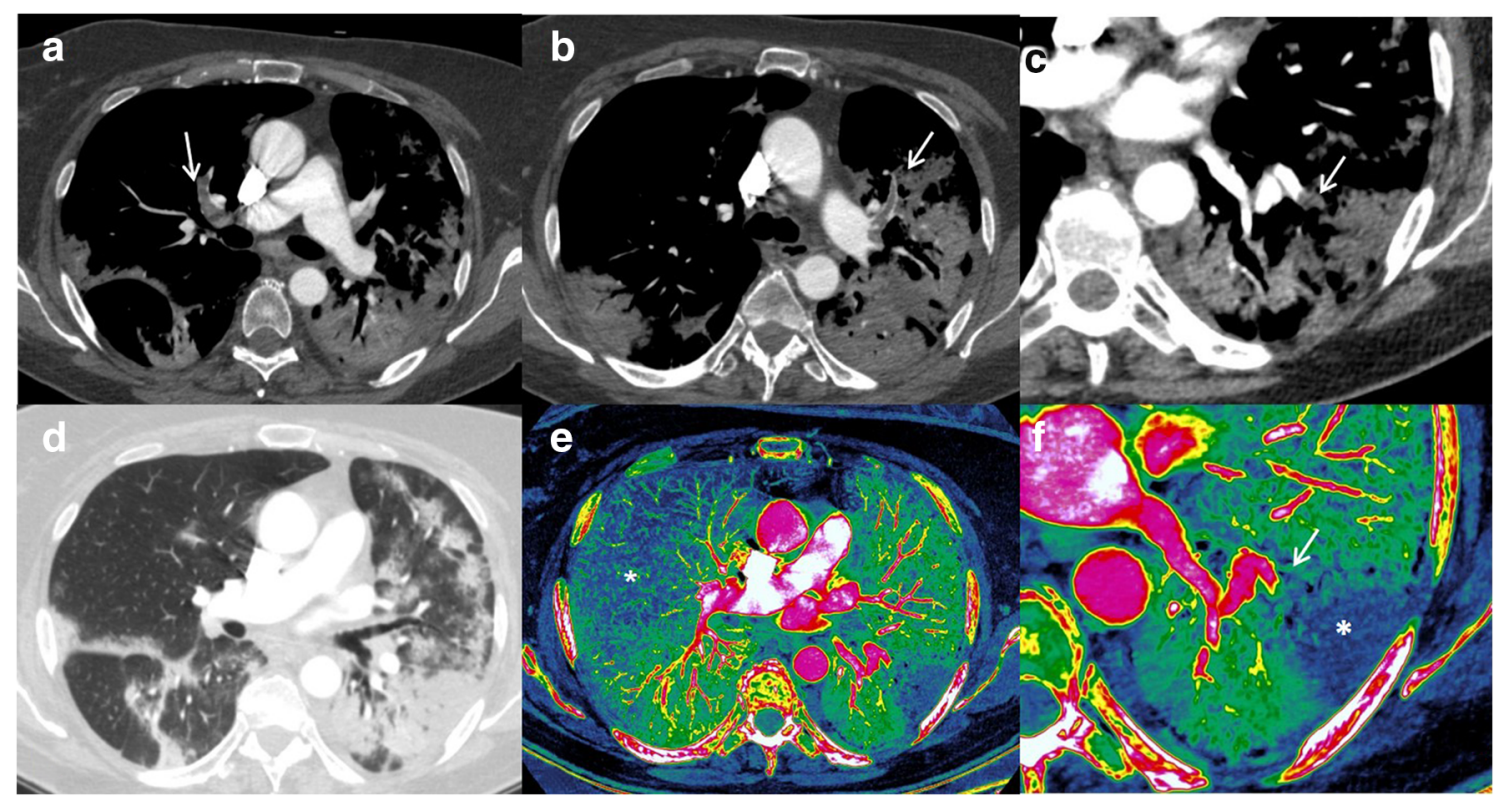

Fig. 5 Bilateral PE with segmental left lower lobe pulmonary infarct over a severe lung involvement. CTPA $(\mathbf{a}, \mathbf{b}, \mathbf{c})$ shows bilateral thrombi (arrows), one located in the distal portion of a segmental artery of the left lower lobe (c). Pulmonary window image (d) depicts multiple GGO areas and consolidations, with typical peripheral sparing consistent with
COVID-19 lung involvement. Iodine map images (e and f) allow us to define right lung hypoperfusion (e; asterisk) and a triangular hypoperfused lesion (f; asterisk), inside the extensive lung involvement and distal to the arterial thrombus, representing a pulmonary infarct 
detect distal involvement by depicting hypoperfused areas in the iodine maps (Figs. 2, 4, and 5). However, in this series, we did not monitor the number of cases diagnosed only by DECT, as both CTPA and DECT were used to establish PE diagnosis. Even though no studies were considered "non-diagnostic", some subsegmental defects may have remained undetected due to respiratory motion artefacts. A recent study by Idilman et al [17] also suggested that DECT analysis might depict perfusion deficits in mild COVID-19 patients that would otherwise be missed with conventional CT angiography. Additional imaging testing, such as perfusion SPECT/CT or DVT ultrasound as a thrombosis screening test, could also be implemented in these patients [10] to increase the certainty of $\mathrm{PE}$ and to complement clinical evaluation. It is also important to draw attention to the fact that several patients $(n=18)$, even though they had COVID-19-compatible CT findings, were excluded from this study because the RT-PCR results were negative or inconclusive. The low sensitivity of RT-PCR has been an added obstacle for identifying COVID-19 patients.

Nevertheless, given the results, there are some highlights to underline: the presence of PE may seem to increase the risk of ICU admission in COVID-19 patients, despite some limitations that will be addressed later. Differences observed in the highest D-dimer values could imply that sequential D-dimer testing could improve the early diagnosis of PE in these patients. Since D-dimer elevation is a common finding among COVID-19 patients [4], further studies are needed to establish a detection cut-off value. This would avoid unnecessary radiation and infection risk to healthy individuals during intrahospital patient transport [18]. This study could also raise an initial recommendation referring to necessary IL-6 testing at hospital admission for acute monitoring of patients with higher determinations, as it could indicate worse clinical evolution, while on its own it does not seem to correlate with PE probability.

There were no significant differences in the probability of PE between anticoagulated patients and those receiving no treatment before CT. No significant differences either were found regardless of the anticoagulation doses received. However, due to the limited sample, the absence of differences between central or lobar PE and segmental or subsegmental PE regarding anticoagulant dosage is difficult to evaluate and could be a starting point for upcoming studies. Further investigation about antithrombotic prophylaxis with a larger sample is also required to assess risk benefit and optimum dosages. As stated by Tang et al [11], the selection of patients to receive anticoagulant treatment should be selective and supported by evidence, and the need to establish a cut-off value for the change in anticoagulant regimen is primarily required. Attention and follow-up of these patients should be taken into account as haemorrhagic events have been described among COVID-19 patients [19].
It is important to remark on the limitations of the presented results. First, the sample size of examination is moderately scarce; therefore, it is intended to raise the alarm about thrombotic events in a COVID-19 scenario and provide a source for future studies with larger samples. The retrospective nature of the study could only show an association but not determine correlation: it is unclear whether PE is a result of severe presentation of COVID-19, as disease extent (score) was similar in both PE and non-PE groups. IL-6 determination on admission as a biomarker of proinflammatory state was not significantly different between these groups. These results suggest that PE may be unrelated to the severity of COVID-19. Gervaise et al [20] also found no differences in the severity of pulmonary findings in CT and suggest the association between APE and non-severe and non-hospitalised COVID-19 patients. However, PE presence alone seems to increase the chance of ICU admission $(68.8 \%$ vs $38.7 \%$ in the negative CTPA group), which should be confirmed with prospective studies with larger sample sizes. However, due to the design of this study, it is not possible to establish whether PE was present before or after ICU admission. Other risk factors for PE such as body mass index, recent surgery, comorbidities, or subjacent malignant disease were not controlled and could induce bias.

The highest frequency of involvement of the right lung is possibly representative of a larger COVID-19 population despite the small sample, as blood flow distribution in the supine and prone positions is higher to the right lung [21]. On the other hand, the upper segmental predominance could be the result of the sample size. Despite these limitations, the patients recruited for this study were exclusively confirmed COVID-19 patients with clinical suspicion of PE. These restrictive inclusion criteria lead to a more homogeneous sample that was thoroughly examined and provided important outcomes for future studies.

\section{Conclusion}

PE in COVID-19 patients is a problematic issue that, in our series, predominantly seems to affect segmental arteries and the right lung, especially its upper lobe. However, this statement is based in a small sample of individuals and therefore needs to be approached in future research. The results also suggest that the higher the D-dimer concentration, the greater the likelihood of $\mathrm{PE}$, an assumption that might be assessed in future studies with a larger sample size. Moreover, PE could increase the probability of ICU admission even though it might not be linked with the severity of COVID-19 pneumonia. 
Funding The authors state that this work has not received any funding.

\section{Compliance with ethical standards}

Guarantor The scientific guarantor of this publication is Flavio Zuccarino.

Conflict of interest The authors of this manuscript declare no relationships with any companies whose products or services may be related to the subject matter of the article.

Statistics and biometry Guillem Casamayor kindly provided statistical advice for this manuscript.

No complex statistical methods were necessary for this paper.

Informed consent Written informed consent was waived by the Institutional Review Board.

Ethical approval Institutional Review Board approval was obtained.

\section{Methodology}

- retrospective

- cross sectional study

- performed at one institution

\section{References}

1. Prokop M, van Everdingen W, van Rees Vellinga T et al (2020) CO-RADS - a categorical CT assessment scheme for patients with suspected COVID-19: definition and evaluation. Radiology. https:// doi.org/10.1148/radiol.2020201473:201473

2. Xie X, Zhong Z, Zhao W, Zheng C, Wang F, Liu J (2020) Chest CT for typical 2019-nCoV pneumonia: relationship to negative RTPCR testing. Radiology. https://doi.org/10.1148/radiol. 2020200343

3. Ai T, Yang Z, Hou H et al (2020) Correlation of chest CT and RTPCR testing in coronavirus disease 2019 (COVID-19) in China: a report of 1014 cases. Radiology. https://doi.org/10.1148/radiol. 2020200642

4. Huang C, Wang Y, Li X et al (2020) Clinical features of patients infected with 2019 novel coronavirus in Wuhan, China. Lancet 395:497-506

5. Bosson JL, Barro C, Satger B, Carpentier PH, Polack B, Pernod G (2005) Quantitative high D-dimer value is predictive of pulmonary embolism occurrence independently of clinical score in a welldefined low risk factor population. J Thromb Haemost 3:93-99

6. Crawford F, Andras A, Welch K, Sheares K, Keeling D, Chappell FM (2016) D-dimer test for excluding the diagnosis of pulmonary embolism. Cochrane Database Syst Rev. https://oi.org/10.1002/ 14651858.CD010864.pub2

7. Gong J, Dong H, Xia SQ et al (2020) Correlation analysis between disease severity and inflammation-related parameters in patients with COVID-19 pneumonia. medRxiv. https://doi.org/10.1101/ 2020.02.25.20025643

8. Chen X, Zhao B, Qu Y et al (2020) Detectable serum SARS-CoV-2 viral load (RNAaemia) is closely correlated with drastically elevated interleukin 6 (IL-6) level in critically ill COVID-19 patients. Clin Infect Dis. https://doi.org/10.1093/cid/ciaa449

9. Ulhaq ZS, Soraya GV (2020) Interleukin-6 as a potential biomarker of COVID-19 progression. Med Mal Infect 50:382-383

10. Zhang Y, Zhang Z, Wei R et al (2020) IL (interleukin)-6 contributes to deep vein thrombosis and is negatively regulated by miR-338-5p. Arterioscler Thromb Vasc Biol 40:323-334

11. Tang N, Bai H, Chen X, Gong J, Li D, Sun Z (2020) Anticoagulant treatment is associated with decreased mortality in severe coronavirus disease 2019 patients with coagulopathy. J Thromb Haemost. https://doi.org/10.1111/jth.14817

12. Klok FA, Kruip MJHA, van der Meer NJM et al (2020) Incidence of thrombotic complications in critically ill ICU patients with COVID-19. Thromb Res. https://doi.org/10.1016/j.thromres.2020. 04.013

13. Geyer LL, Scherr M, Körner M et al (2012) Imaging of acute pulmonary embolism using a dual energy CT system with rapid kVp switching: initial results. Eur J Radiol 81:3711-3718

14. Bernheim A, Mei X, Huang $M$ et al (2020) Chest $C T$ findings in coronavirus disease-19 (COVID-19): relationship to duration of infection. Radiology. https://doi.org/10.1148/radiol.2020200463

15. British Society of Thoracic Imaging (2020) Thoracic imaging in COVID 19 infection. Guidance for the Reporting Radiologist (2nd version). Available via https://www.bsti.org.uk/media/ resources/files/BSTI COVID-19 Radiology Guidance version 2_16.03.20.pdf. Accessed 20 March 2020

16. Caruso D, Zerunian M, Polici M et al (2020) Chest CT features of COVID-19 in Rome, Italy. Radiology. https://doi.org/10.1148/ radiol.2020201237

17. Idilman IS, Dizman GT, Duzgun SA et al (2020) Lung and kidney perfusion deficits diagnosed by dual-energy computed tomography in patients with COVID-19 related systemic microangiopathy. Eur Radiol. https://doi.org/10.1007/s00330-020-07155-3

18. Rubin GD, Ryerson CJ, Haramati LB et al (2020) The role of chest imaging in patient management during the COVID-19 pandemic: a multinational consensus statement from the Fleischner Society. Chest. https://doi.org/10.1016/j.chest.2020.04.003

19. Zhang B, Zhou X, Qiu Y et al (2020) Clinical characteristics of 82 death cases with COVID-19. medRxiv. https://doi.org/10.1101/ 2020.02.26.20028191

20. Gervaise A, Bouzad C, Peroux E, Helissey C (2020) Acute pulmonary embolism in non-hospitalized COVID-19 patients referred to CTPA by emergency department. Eur Radiol. https://doi.org/10. 1007/s00330-020-06977-5

21. Wieslander B, Ramos JG, Ax M, Petersson J, Ugander M (2019) Supine, prone, right and left gravitational effects on human pulmonary circulation. J Cardiovasc Magn Reson 21:69

Publisher's note Springer Nature remains neutral with regard to jurisdictional claims in published maps and institutional affiliations. 